\title{
Editorial
}

\section{Kidney Attack: Overdiagnosis of Acute Kidney Injury or Comprehensive Definition of Acute Kidney Syndromes?}

\author{
Claudio Ronco
}

Department of Nephrology, Dialysis and Transplantation, International Renal Research Institute (IRRIV), San Bortolo Hospital, Vicenza, Italy

Acute kidney injury (AKI) is today facing a complex phase of characterization due to a lack of an accepted definition for a long time. The same problem occurred in the past to the acute coronary syndrome (ACS). Acute myocardial infarction (AMI) has undergone a long process over the years before finding a standardization of diagnostic criteria and a rationale classification. ACS are well defined by a screening process based on symptoms (chest pain at onset), EKG abnormalities (STEMI: ST-elevation myocardial infarction and NSTEMI: non-ST elevation myocardial infarction) and biomarkers such as troponins (NSTEMI vs. renal angina). All these clinical pictures are somehow falling under the umbrella term of 'heart attack'. Different names have been used to define a similar disorder for the kidney (acute tubular necrosis, acute renal failure, acute kidney disease) and although the commonest term is acute kidney injury/AKI, recently a consensus has been developed to call this syndrome 'kidney attack' $[1,2]$. For the kidney, authors agree that the pathophysiology can be extremely complex and well beyond a single ischemic insult (toxic, allergic, metabolic, obstructive, septic). The final effect can be an acute structural damage (often tubular) and/or an acute dysfunction or both. There is an effort today to increase alertness and awareness about AKI; the term 'kidney attack' may recall what occurs in the heart with its severe consequences and may contribute to a better understanding of this dangerous condition. In spite of the multiple causes and the complex pathophysiology, so far we have only relied on one single parameter to diagnose AKI, i.e. serum creatinine. In the literature the reported incidence and mortality for AKI ranges from 1 to $31 \%$ while mortality ranges from 28 to $82 \%$. This depends on the used definitions since diagnostic criteria and severity of the syndrome strongly affects both epidemiology and outcome [3]. While in the past, terms such as acute tubular necrosis (ATN) or acute renal failure (ARF) were mostly descriptive and they were never based on a specific biochemical syntax, today AKI is graded in terms of severity using both serum creatinine and urine output as criteria for the diagnosis. This has been the advance produced by ADQI with the creation of RIFLE, subsequently modified by AKIN stages and finally twigged by KDIGO guidelines [3, 4].

The term 'kidney attack' has no biochemical reference nor does it grade the severity of the insult, but it stresses the concept that while heart attack is documented or suspected by the presence of chest pain and EKG abnormalities, the kidney is a silent organ and clinical evidence for this disorder may be scanty although a severe condition may develop [1]. Furthermore, although clinically silent

\section{KARGER}

E-Mail karger@karger.com

www.karger.com/bpu
(C) 2013 S. Karger AG, Basel

0253-5068/13/0362-0065\$38.00/0
Prof. Claudio Ronco

Department of Nephrology, Dialysis and Transplantation

International Renal Research Institute (IRRIV), San Bortolo Hospital

Viale Rodolfi 37, IT-36100 Vicenza (Italy)

E-Mail cronco@goldnet.it 


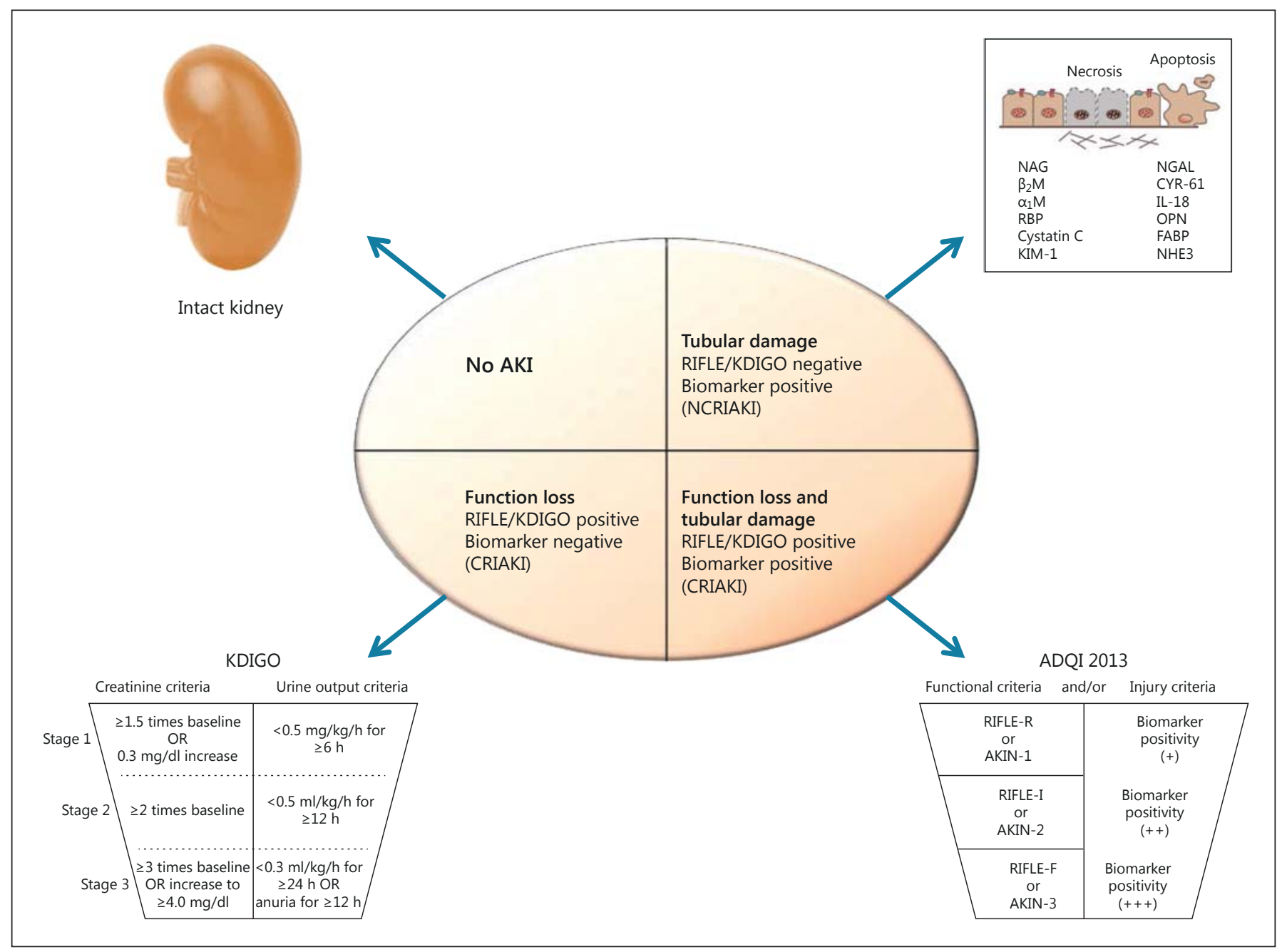

Fig. 1. New criteria for AKI diagnosis are displayed on the oval shape. Upper left sector: no AKI with an intact kidney; other three sectors: in order to diagnose AKI selecting the worst criterion [function (RIFLE/AKIN) or damage] is recommended. Upper right sector: new damage biomarkers positivity allow to define NCRIAKI (non-creatinine increase AKI) or subclinical AKI.

or 'subclinical', one or multiple kidney attacks may have an important effect on both short- and long-term clinical outcomes and kidney function [5-8].

In practice, the diagnosis of AKI has so far been made based on changes in serum creatinine or urine output, confounding de facto kidney injury and kidney dysfunction. Urine output may be subject to changes due to several causes and creatinine levels can also vary widely depending on a large number of non-renal factors (e.g. age, gender, muscle mass, hydration status, etc.). This observation underlines the inadequacy of these two parameters to describe the nature of the syndrome, to characterize its
Lower left sector: RIFLE/KDIGO functional parameters (urine output and creatinine) are utilized to diagnose and stage AKI with functional loss. Lower right sector: AKI with functional loss and tubular damage can be diagnosed both with functional criteria and damage criteria according to the most recent ADQI consensus. severity and to include all the nuances of the kidney attack. Because of a large renal reserve, up to $50 \%$ of kidney function may be lost before serum creatinine rises. Furthermore, creatinine half-life is quite long and its variations over time are slow. Thus, serum creatinine does not accurately depict kidney function until a new steady state has been achieved after GFR has changed (up to 2-3 days after injury and constant GFR alteration). In a recent ADQI consensus, a new perspective has been suggested for the diagnosis of AKI (or kidney attack) including a new category of kidney disorders defined by positivity of damage biomarkers and negativity of creatinine or urine 


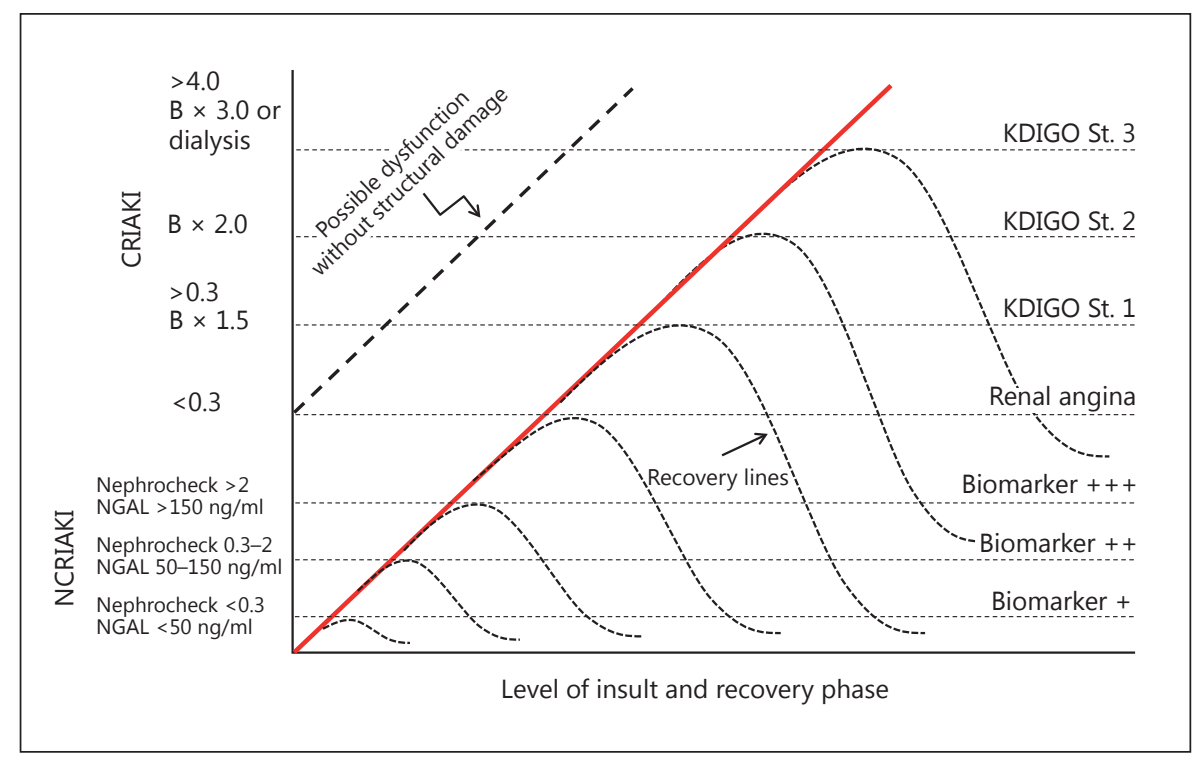

Fig. 2. Various categories of AKI are displayed according to damage criteria and functional criteria. For damage criteria, there are currently insufficient injury biomarker data to support staging of AKI, however the semiquantitative trend for increasing biomarker severity associated with increasing kidney damage is suggested by the literature $[8,11,12]$, for some biomarkers such as plasma NGAL and cell cycle arrest biomarkers (Nephrocheck: insulin-like growth factor-binding protein 7 and tissue inhibitor of metalloproteinase-2). When function loss begins, there is an intermediate phase in which creatinine rises but below the threshold level of 0.3 $\mathrm{mg} / \mathrm{dl}$. This phase is defined in the appropriate clinical setting as RA. Until this point the syndrome is subclinical and defined as NCRIAKI. When function loss progresses, creatinine increases beyond $0.3 \mathrm{mg} / \mathrm{dl}$ and the syndrome becomes clinically evident (CRIAKI) being staged by the RIFLE/KDIGO criteria. For every point, damage and dysfunction may progress towards the more severe phase, or proceed towards a recovery phase with limitation of the damage and partial or full restoration of function (NGAL: Alere, USA; Nephrocheck, Astute Medical, USA). output criteria (fig. 1) $[3,9,10]$. Furthermore, a new entity has also recently been described called renal angina (RA), characterizing those patients who display a slight increase in serum creatinine under the critical value of 0.3 $\mathrm{mg} / \mathrm{dl}$ so far utilized as the biochemical threshold to diagnose AKI [9]. The dramatic importance of these new categories of AKI is highlighted by the fact that such patients are at risk for developing severe AKI or they are presenting worse outcomes in terms of morbidity and mortality compared to the control population $[5-8,10,11]$.

Thus, a new nomenclature for kidney attack and related syndromes with a wider spectrum of clinical conditions is available today and it should be used for diagnostic purposes (fig. 1). New biomarkers can help us where the symptoms are scanty or absent or when creatinine or urine output are not discriminant. To make a comparison with heart attack, we might parallel creatinine increase AKI (CRIAKI or AKI with dysfunction) with STEMI, and non-creatinine increase AKI (NCRIAKI or AKI with damage but not dysfunction, defined subclinical and characterized by positivity of biomarkers) with NSTEMI.
Between the two situations, we can place RA as a transition syndrome between subclinical AKI and overt clinical AKI, somehow resembling the unstable angina for the heart [1]. As depicted in figure 2, there might be a progression in severity moving from one type to another, but every type can also be an independent condition and result in partial or full recovery. The issue of recovery deserves a further comment. When a kidney attack occurs, a variable number of nephrons may be damaged or destroyed. Specific biochemical pathways are activated to limit the level of damage as in the case of cell cycle arrest. In other cases, repair mechanisms are immediately put to work, although local conditions and the presence or absence of biochemical mediators may lead to a full, partial or even maladaptive repair. In some cases, the damage may be so important that locally, nephrons are replaced by fibrotic tissue thus reducing the functioning nephron mass of the individual $[12,13]$. This damage becomes clinically evident only when more than $50 \%$ of the nephron mass is affected and the renal functional reserve (RFR) has been completely lost. For less severe damage 
situations, the individual may undergo a progressive nephron loss without any clinical signs or symptoms. This, however, has a profound impact on the long-term outcome, increasing the susceptibility of the kidney with a higher risk to develop clinical AKI even in the presence of a moderate insult or exposure, and/or progression to chronic kidney disease.

In conclusion, AKI or kidney attack seems to be a syndrome with a level of complexity well beyond what was described or so far suspected. A thorough campaign for awareness and alertness should be undertaken to prevent kidney attack and to make an early diagnosis of any type of kidney damage including the subclinical forms. As it is commonly accepted for heart attack to include STEMI, NSTEMI and unstable angina in the diagnosis of ACS, the same should be done for CRIAKI, NCRIAKI and RA.
Is there a risk to overdiagnose the syndrome? The same question was posed when the five grades of chronic kidney disease were proposed by KDOQI and today we know very well that this has been a major advancement in terms of early referral, prevention of progression and therapeutic strategies. We think that new kidney attack diagnostic criteria do not carry the risk of overdiagnosis of AKI, but rather they help to unveil what has been neglected or underestimated for too long simply because it was not clinically detectable. We want to preserve the nephrons one by one since we feel that every single unit is important for the overall function and for the longterm destiny of the kidney and the individual.

\section{References}

1 Ronco C, Chawla LS, McCullough PA: Kidney attack vs. heart attack: evolution of classification and diagnostic criteria. Lancet, in press.

$\checkmark 2$ Kellum JA, Bellomo R, Ronco C: Kidney attack. JAMA 2012;307:2265-2266.

$\checkmark 3$ McCullough PA, Shaw AD, Haase M, Bouchard J, Waikar SS, Siew ED, Murray PT, Mehta RL, Ronco C: Diagnosis of Acute Kidney Injury Using Functional and Injury Biomarkers: Workgroup Statements from the Tenth Acute Dialysis Quality Initiative Consensus Conference. Contrib Nephrol. Basel, Karger, 2013, vol 182, pp 13-29.

4 KDIGO Clinical Practice Guideline for Acute Kidney Injury. Kidney Int 2012;2:1-141.

$\checkmark 5$ Ronco C, Kellum JA, Haase M: Subclinical AKI is still AKI. Crit Care 2012;16:313.

-6 Haase M, Kellum JA, Ronco C: Subclinical AKI - an emerging syndrome with important consequences. Nat Rev Nephrol 2012;8:735-739.
7 Ronco C, Stacul F, McCullough PA: Subclinical acute kidney injury (AKI) due to iodinebased contrast media. Eur Radiol 2013;23: 319-323.

8 Haase M, Devarajan P, Haase-Fielitz A, Bellomo R, Cruz DN, Wagener G, Krawczeski CD, Koyner JL, Murray P, Zappitelli M, Goldstein SL, Makris K, Ronco C, Martensson J, Martling CR, Venge P, Siew E, Ware LB, Ikizler TA, Mertens PR: The outcome of neutrophil gelatinase-associated lipocalin-positive subclinical acute kidney injury: a multicenter pooled analysis of prospective studies. J Am Coll Cardiol 2011;57:1752-1761.

9 Goldstein SL, Chawla LS: Renal angina. Clin J Am Soc Nephrol 2010;5:943-949.

10 McCullough PA, Bouchard J, Waikar SS, Siew ED, Endre ZH, Goldstein SL, Koyner JL, Macedo E, Doi K, Di Somma S, Lewington A, Thadhani R, Chakravarthi R, Ice C,
Okusa MD, Duranteau J, Doran P, Yang L, Jaber BL, Meehan S, Kellum JA, Haase M, Murray PT, Cruz D, Maisel A, Bagshaw SM, Chawla LS, Mehta RL, Shaw AD, Ronco C: Implementation of Novel Biomarkers in the Diagnosis, Prognosis, and Management of Acute Kidney Injury: Executive Summary from the Tenth Consensus Conference of the Acute Dialysis Quality Initiative (ADQI). Contrib Nephrol. Basel, Karger, 2013, vol 182 , pp 5-12.

11 Ronco C, Ricci Z: The concept of risk and the value of novel markers of acute kidney injury. Crit Care 2013;17:117.

12 Ronco C, Chawla LS: Acute kidney injury: kidney attack must be prevented. Nat Rev Nephrol 2013;9:198-199.

13 Ronco C, Rosner MH: Acute kidney injury and residual renal function. Crit Care 2012; 16:144. 